\title{
Effect of Hypoxic-Ischemic Injury on Serine Palmitoyltransferase Activity in the Developing Rat Brain
}

\author{
Masaki Daigo $^{\text {a }}$ Yasuhiro Arai $^{\mathrm{a}}$ Kyoichi Oshida $^{\mathrm{b}}$ Yohei Kitamura $^{\mathrm{d}}$

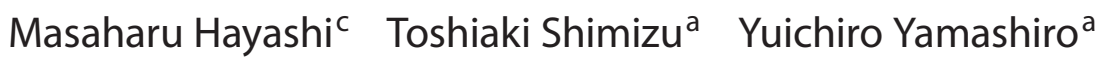 \\ ${ }^{a}$ Department of Pediatrics, Juntendo University School of Medicine, ${ }^{b}$ Department of Nutrition, Kemin Co. Ltd., and \\ 'Department of Clinical Neuropathology, Tokyo Metropolitan Institute for Neuroscience, Tokyo; ${ }^{\mathrm{d}}$ Nutritional Science \\ Laboratory, Morinaga Milk Industry Co. Ltd., Zama, Japan
}

\section{Key Words}

Myelination $\cdot$ Serine palmitoyltransferase $\cdot$ Proteolipid protein $\cdot$ Myelin basic protein $\cdot$ Hypoxic-ischemic brain injury

\begin{abstract}
Background: Sphingolipid metabolism is strongly associated with central nervous system myelination. Ceramide is the most active of the sphingolipid metabolites. On the basis of ceramide biosynthesis indicated by serine palmitoyltransferase activity and cerebroside generated by ceramide, the evaluation of serine palmitoyltransferase activity in developing brains or hypoxic-ischemic damaged brains is worthwhile. Methods: Using a scintillation counter, we assessed serine palmitoyltransferase activity, a rate-limiting enzyme of sphingolipid metabolism, in the brains of developing rats and compared the activity with hypoxic-ischemic brains, using the method of Rice on postnatal day 7 (P7). Results: In the control groups, serine palmitoyltransferase activity was detected in the microsomal fraction of whole brain homogenates from $\mathrm{P} 4$, which was earlier than the initial expression of myelin-specific proteins such as myelin basic protein and proteolipid protein on immunochemistry. Serine palmitoyltransferase activity increased along with development on P8, P10, P14 and P21. However, hypoxic-ischemic brains
\end{abstract}

showed lower serine palmitoyltransferase activity than control brains on P8, P10, P14 and P21. Conclusions: These results suggest that increase in serine palmitoyltransferase activity before myelin-specific protein expression may be an initial step in myelinogenesis and a decline in serine palmitoyltransferase activity in hypoxic-ischemic brains may be one of the major causes of delayed myelination.

Copyright $\odot 2008$ S. Karger AG, Basel

\section{Introduction}

Periventricular leukomalacia (PVL) is caused by an ischemic infarction of the cerebral white matter adjacent to the lateral ventricles, especially in premature infants born before 35 weeks of gestation [1]. It is known that PVL affects $26 \%$ of premature infants with a birth weight $<1,500$ g and has increased along with a reduction in the mortality of these infants. The typical clinical manifestation of PVL is cerebral palsy [2]. Although the mechanism underlying PVL is not clear, it is thought that high vulnerability of the cerebral white matter to ischemic infarction in premature infants is one of the major causes. It has been suggested that the progenitor cells of oligodendrocytes are easily damaged by oxidative stress and excitotoxicity. Recently, selective head cooling therapy

\section{KARGER \\ Fax +41613061234 E-Mail karger@karger.ch} www.karger.com
() 2008 S. Karger AG, Basel $1015-2008 / 08 / 0756-0330 \$ 24.50 / 0$

Accessible online at:

www.karger.com/pat
Masaki Daigo

Department of Pediatrics, Juntendo University School of Medicine

3-1-1, Hongo, Bunkyo-ku

Tokyo, 113-8421 (Japan)

Tel. +81 33813 3111, Fax +81 55948 3215, E-Mail bigfive197322@yahoo.co.jp 
has been tried in infants with hypoxic-ischemic injury [3], but its efficacy has not been fully established.

The major pathological feature of PVL is chronic disturbance of myelination and it has been suggested that oligodendrocyte progenitors are a target of ischemic injury [4]. Myelin is uniquely found in multilamellar membranes in the central nervous system (CNS) and it has a high lipid content, being composed of a lipid bilayer (particularly sphingolipids such as sphingomyelin, cerebroside and sulfatide) combined with proteins such as myelin basic protein (MBP) and proteolipid protein (PLP) [5].

We have reported that dietary sphingomyelin contributes to CNS myelination in developing rats [6]. De novo synthesis of sphingolipids is initiated by serine palmitoyltransferase (SPT), which is the rate-limiting enzyme for the reaction by which serine and palmitoyl-ConA form 3-ketosphinganine (fig. 3) [6-8]. SPT is located in the endoplasmic reticulum and the Golgi apparatus [6]. Cerebroside is synthesized from ceramide that is derived from sphingomyelin or else undergoes de novo synthesis by SPT, and is recognized as one of the biomarkers of myelination $[9,10]$. It has been demonstrated that synthesis of sphingolipids and their metabolites is necessary for dendritic growth and survival of cerebellar Purkinje cells [11], but whether hypoxic-ischemic injury affects sphingolipid biosynthesis has remained obscure.

In this study, we assessed the developmental changes of SPT activity and compared the activity of this enzyme between controls and hypoxic-ischemic brains of rats to clarify the correlation of SPT activity with myelination or hypoxic-ischemic insult.

SPT activity and immunohistochemical evidence of white matter damage in developing rat brains were examined by using the hypoxic-ischemic rat model previously reported by Rice et al. [12].

\section{Materials and Methods}

\section{Study Design}

All procedures for the care and use of animals conformed to the Guidelines for Animal Experiments of the Juntendo University School of Medicine. Unsexed Wistar rats were purchased on postnatal day 3 (P3) together with their dams from Charles River Laboratories Japan Inc. (Kanagawa, Japan). The animals were housed in polycarbonate cages in a room with controlled temperature $\left(25^{\circ} \mathrm{C}\right)$ and lighting time $(08.00$ to $20.00 \mathrm{~h})$. Forty-five pups were randomly assigned to 2 groups, a control group $(\mathrm{n}=25)$ and a Rice model group $(n=20)$. Pups in both groups were suckled by their dams and allowed free access to a commercial diet (CRF-1; Oriental Yeast Co. Ltd., Tokyo, Japan) and water after the suckling period until each pup was sacrificed.
Rice Model

Neonatal ischemic-hypoxic injury was created by the method of Rice et al. [12]. Briefly, P7 Wistar rats were anesthetized with isoflurane and the left common carotid artery was ligated using 4- 0 surgical silk, followed by breathing $8 \%$ oxygen for $1.5 \mathrm{~h}$ in a heated box at $37^{\circ} \mathrm{C}$. After the procedure, the pups were returned to their dam and reared normally until decapitation. Control rats did not undergo surgery or exposure to hypoxia.

\section{Chemicals}

DL-erythrodihydrosphingosine, HEPES, EDTA, palmitoylConA, DTT, L-serine and pyridoxal 5'-phosphate were all purchased from Sigma-Aldrich Japan (Tokyo, Japan). Radioactive L- $\left[3-{ }^{14} \mathrm{C}\right]$ serine was obtained from Amersham Bioscience (Tokyo, Japan). All other chemicals and solvents used were of analytical grade.

\section{Antibodies}

Antisera were used for immunohistochemistry. Polyclonal antibodies directed against MBP or platelet-derived growth factor receptor (PDGF)- $\alpha$ were purchased from Nichirei Co. (Tokyo, Japan) or Santa Cruz Biotechnology Inc. (Santa Cruz, Calif., USA). The monoclonal antibody for PLP was a kind gift from Dr. Takashi Yamamura (National Center of Neurology and Psychiatry, Tokyo, Japan) [13].

\section{Immunohistochemistry}

Specimens were fixed in $10 \%$ neutral buffered formalin for approximately $24 \mathrm{~h}$ at room temperature, cut into 3 pieces, embedded in paraffin, and then cut into 5 - $\mu$ m-thick serial sections. After treatment with $0.5 \% \mathrm{H}_{2} \mathrm{O}_{2}$ in methanol and blocking with serum, the sections were incubated overnight at $4^{\circ} \mathrm{C}$ with a polyclonal antibody targeting MBP (prediluted) and PDGF- $\alpha$ (1:500) or a monoclonal antibody for PLP $(1: 1,000)$. Subsequently, the sections were stained by the avidin-biotin peroxidase complex method (Vectastain ABC kit; Vector, Burlingame, Calif., USA). Application of a chromogen (3,3'-diaminobenzidine tetrahydrochloride) was followed by counterstaining with hematoxylin to allow identification of immunoreactivity [14].

\section{Isolation of Microsomes from Whole Brains}

To evaluate SPT activity, the whole brain was removed from Rice model rats sacrificed on P8 $(n=5)$, P10 $(n=5)$, P14 $(n=5)$ and P21 $(n=5)$. Control rats were sacrificed on P4 $(n=5)$ and the same number of rats was killed on each of the other days without hypoxic-ischemic brain damage. The brain was immediately removed from each rat and placed in an ice-cold solution containing $0.32 \mathrm{M}$ sucrose, $5 \mathrm{~mm}$ EDTA and $50 \mathrm{~mm}$ HEPES ( $\mathrm{pH}$ 7.4). Then, the brains were homogenized, and the cell nuclei, debris and mitochondria were removed by centrifugation at $10,000 \mathrm{~g}$ for $15 \mathrm{~min}$ at $4^{\circ} \mathrm{C}$. The microsomal fraction was sedimented at $10,500 \mathrm{~g}$ for $1 \mathrm{~h}$ at $4^{\circ} \mathrm{C}$, washed with a solution containing $50 \mathrm{mM}$ HEPES and $5 \mathrm{mM}$ EDTA, recentrifuged at $10,500 \mathrm{~g}$ for $1 \mathrm{~h}$ at $4^{\circ} \mathrm{C}$, and finally suspended in $50 \mathrm{mM}$ HEPES, $5 \mathrm{~mm}$ EDTA and $5 \mathrm{~mm}$ DTT $(1 \mu \mathrm{l} / \mathrm{mg}$ original tissue) and frozen at $-80^{\circ} \mathrm{C}$. No significant change of SPT activity was found after several months of storage at this temperature. 


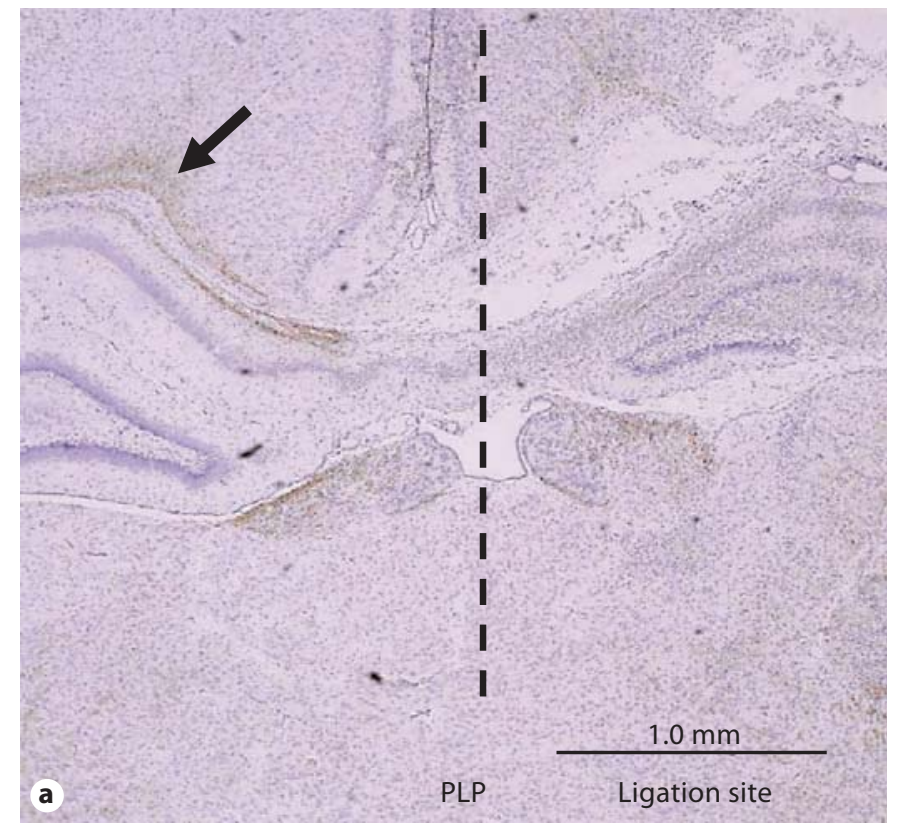

Fig. 1. Immunohistochemical staining for PLP and MBP of hippocampus sections from a P10 rat. The positive fibers are indicated by arrows. In the white matter, PLP (a) and MBP (b) staining is markedly decreased in the ligation site within the ischemic tissue. c Immunohistochemical staining for PDGF- $\alpha$ in a P14 rat. The positive fibers are indicated by open arrows. In the white matter, PDGF- $\alpha$-positive fibers are detected around the ischemic damage foci.

\section{Measurement of the Protein Content and SPT Activity in}

Whole Brain Microsomes

The protein content of the microsomes was measured by the bicinchoninic acid assay (Pierce Biotechnology, Rockford, Ill., USA) [15] using bovine serum albumin as the standard. The SPT activity in whole brain homogenates was analyzed using the method described by Williams et al. [16] with minor modifications. Enzyme activity was measured as the transfer of radioactivity to 3-ketosphinganine, a metabolite of SPT, from L- $\left[3-{ }^{14} \mathrm{C}\right]$ serine. First, L- $\left[3-{ }^{14} \mathrm{C}\right]$ serine was diluted to $370 \times 10^{6} \mathrm{~Bq} / \mathrm{mmol}$ with Lserine. A solution for testing 20 samples was prepared with $100 \mu \mathrm{l}$ of $1 \mathrm{M}$ HEPES, $50 \mu \mathrm{l}$ of $10 \mathrm{mM}$ EDTA, $50 \mu \mathrm{l}$ of $5 \mathrm{~mm}$ DTT and 100 $\mu \mathrm{l}$ of $50 \mu \mathrm{M}$ pyridoxal phosphate. Then, $300-\mu \mathrm{l}$ aliquots were divided into 20 tubes $(15 \mu \mathrm{l} / \mathrm{tube})$ and $150 \mu \mathrm{g}$ of microsomal protein from the whole brain homogenate was accurately added. The reaction was initiated by adding $10 \mu \mathrm{l}$ of $0.16 \mathrm{~mm}$ palmitoyl-ConA and incubation was done at $37^{\circ} \mathrm{C}$ for $20 \mathrm{~min}$ with shaking. Subsequently, the reaction was terminated by adding $0.5 \mathrm{ml}$ of chlo-
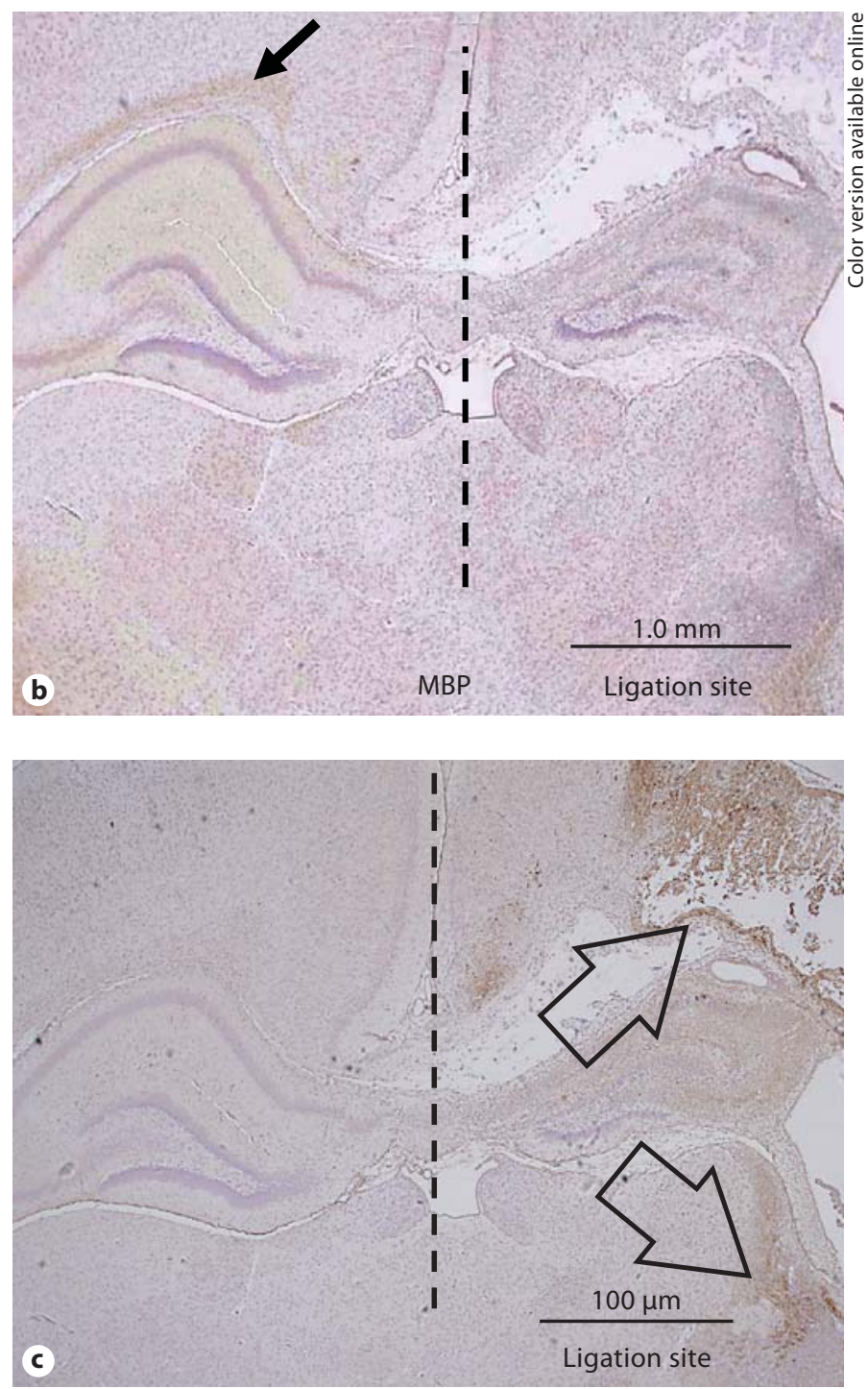

roform and $1.0 \mathrm{ml}$ of methanol. The solution was centrifuged at $3,000 \mathrm{rpm}$ for $10 \mathrm{~min}$, the upper layer was removed, and $2.0 \mathrm{ml}$ of $\mathrm{H}_{2} \mathrm{O}$ was added before centrifuging again under the same conditions. This procedure was repeated twice to completely remove unrecruited L- $\left[3-{ }^{14} \mathrm{C}\right]$ serine from the lower layer. After removing the upper layer, $0.8 \mathrm{ml}$ of the lower layer was transferred to the vial of a liquid scintillation counter and the organic solvent was removed under a stream of nitrogen. Finally, $4 \mathrm{ml}$ of liquid scintillation counter cocktail (Ultima Gold XR; PerkinElmer Japan Co. Ltd., Kanagawa, Japan) was added and the radioactivity was measured with an LSC-6000 scintillation counter (Aloka Co. Ltd., Tokyo, Japan).

\section{Statistics}

All data are expressed as means \pm SEM. Student's $t$ test was utilized to compare mean values between the Rice model group and control group using Microsoft Excel, version 2003 for Windows. 


\section{Results}

\section{Immunohistochemistry}

In macroscopic findings, there were no abnormalities of the gray or white matter in the brains of the control group (from P4 to P21). On the contrary, left-sided mild to moderate cerebral atrophy was recognized in the hypoxic-ischemic brains after ligation of the left common carotid artery. In microscopic findings, among the rats (from P8 to P21) subjected to hypoxic-ischemic injury, eosinophilic neurons were detected in the cerebral cortex and hippocampus of the left hemisphere. In the white matter, ischemic damage was most marked around the lateral ventricle at the left site.

PLP immunoreactivity was detected in myelinated fiber tracts from P10 onwards, while MBP immunoreactivity was observed in the cerebral white matter from P14. Staining for PLP and MBP was markedly decreased in the ischemic brain tissue (fig. 1a, b). The area of axonal injury revealed by reduced PLP or MBP staining was well correlated with the area of neuronal damage as assessed by hematoxylin and eosin staining. PDGF- $\alpha$ immunoreactivity was observed in glial cells around ischemic damaged area from P14 (fig. 1c).

\section{SPT Activity in Whole Rat Brains}

In control brains, SPT activity increased along with development from P4 to P21 (fig. 2). In the Rice model group, SPT activity decreased transiently until P14, but then increased again from P14 to P21. At 1 day after the hypoxic-ischemic insult (P8), SPT activity was lower in the Rice model group than in the control group. By 3 days after the insult (P10), SPT activity was significantly lower in the Rice model group compared with the control group $(\mathrm{p}=0.048)$, while there was no statistical difference of SPT activity between the groups on P21 $(\mathrm{p}=0.53)$.

\section{Discussion}

SPT is the rate-limiting enzyme for de novo synthesis of sphingolipids [7]. Ceramide is the most active of the sphingolipid metabolites, while cerebroside is generated from ceramide by ceramide galactosyltransferase, which is the key enzyme to ceramide (fig. 3) [17]. Because the period of maximum cerebroside biosynthesis corresponds with the time when myelination is most active [18], cerebroside is generally recognized as universal marker of CNS myelination $[19,20]$. In rats, cerebroside is almost undetectable in the brain before P10, but the

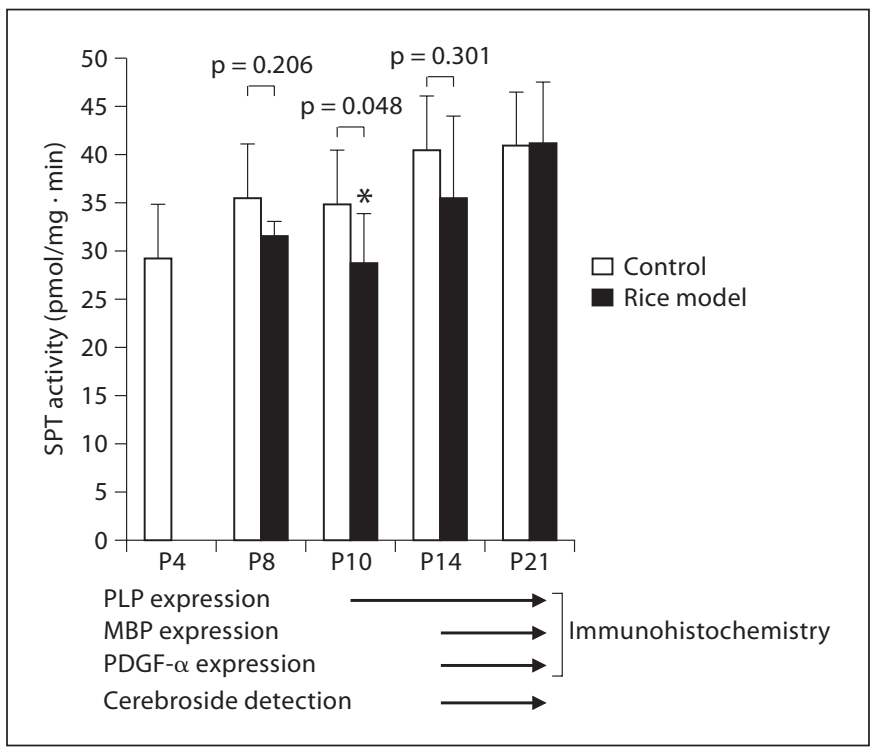

Fig. 2. Changes of whole brain SPT activity in the control and Rice model groups during development. SPT activity was detected from P4. PLP expression was detected from P10, MBP and PDGF- $\alpha$ from P14 with the immunohistochemical method. Cerebroside detection was from P14. Data are presented as means \pm SE. * Significant difference at $\mathrm{p}<0.05$.

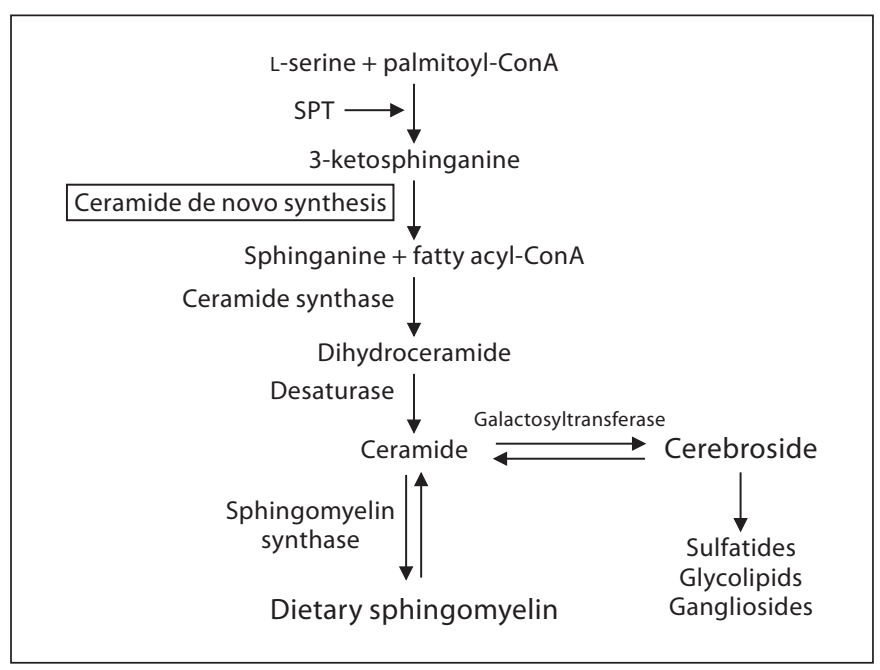

Fig. 3. Metabolic pathway of sphingolipids.

cerebroside content increases markedly from P14 [18]. In the present study, the ceramide biosynthesis indicated by SPT activity commenced on P4, earlier than cerebroside detection or MBP and PLP expression were detectable with the immunohistochemical method. Along with the development from P4 to P21, the activity increased in 
controls. On the basis of these results, an increment of SPT activity before myelination may be an initial step in myelinogenesis.

It is generally recognized that the normal rat brain at P7 corresponds to the human brain at term [22]. We created a modified Rice model by exposing Wistar rats to hypoxia and carotid artery ligation on P7 [12]. Several studies have already been conducted with this model to examine acute changes of neuronal biochemical parameters and neurotransmitters [23-25]. Our experiment confirmed that pathological changes in the Rice model, such as the decrease in white matter volume or MBP and PLP expression, approximately corresponded to those of human PVL. The present study also showed that the SPT activity of brains subjected to hypoxic-ischemic injury was lower than normal on P8, P10 and P14 (fig. 2).

This may mean that the decline in ceramide biosynthesis indicated by SPT activity may cause delayed my- elination. On the assumption that PDGF- $\alpha$ favors the formation of glial or oligodendrocyte progenitor cells [26], the increase in SPT activity according to PDGF- $\alpha$ expression from P14 to P21 in the hypoxic-ischemic group may mean reperfusion of vessels or recanalization of axonal flow around damaged tissue. Both during development and remyelination, newly synthesized cerebroside is accumulated [9]. On the basis of ceramide biosynthesis indicated by SPT activity, the increase in SPT activity in the hypoxic-ischemic group may play an important role for remyelination.

\section{Acknowledgements}

We thank Dr. Akihito Takei (Tokyo Medical College) for technical assistance with the Rice model.

\section{References}

1 Volpe JJ: Brain injury in the premature infant: overview of clinical aspects, neuropathology, and pathogenesis. Semin Pediatr Neurol 1998;5:135-151.

-2 Kuban KC, Leviton A: Cerebral palsy. N Engl J Med $1994 ; 330: 188-195$.

3 Gunn AJ: Cerebral hypothermia for prevention of brain injury following perinatal asphyxia. Curr Opin Pediatr 2000;12:111-115.

$\$ 4$ Volpe JJ: Brain injury in the premature infant - from pathogenesis to prevention. Brain Dev 1997; 19:519-534

5 Porter BE, Tennekoon G: Myelin and disorders that affect the formation and maintenance of this sheath. Ment Retard Dev Disabil Res Rev 2000;6:47-58.

6 Oshida K, Shimizu T, Takase M, Tamura Y, Shimizu T, Yamashiro Y: Effects of dietary sphingomyelin on central nervous system myelination in developing rats. Pediatr Res 2003;53:589-593.

7 Luberto C, Hannun YA: Sphingolipid metabolism in the regulation of bioactive molecules. Lipids 1999;34(suppl):S5-S11.

-8 Perry DK, Carton J, Shah AK, Meredith F, Uhlinger DJ, Hannun YA: Serine palmitoyltransferase regulates de novo ceramide generation during etoposide-induced apoptosis. J Biol Chem 2000;275:9078-9084.

-9 Jurevics H, Hostettler J, Muse ED, Sammond DW, Matsushima GK, Toews AD, Morell P: Cerebroside synthesis as a measure of the rate of remyelination following cuprizoneinduced demyelination in brain. J Neurochem 2001;77:1067-1076.
10 Muse ED, Jurevics H, Toews AD, Matsushima GK, Morell P: Parameters related to lipid metabolism as markers of myelination in mouse brain. J Neurochem 2001;76:77-86.

11 Furuya S, Ono K, Hirabayashi Y: Sphingolipid biosynthesis is necessary for dendrite growth and survival of cerebellar Purkinje cells in culture. J Neurochem 1995;65:15511561

12 Rice JE, Vannucci RC, Brierley JB: The influence of immaturity on hypoxic-ischemic brain damage in the rat. Ann Neurol 1981;9: 131-141.

13 Yamamura T, Konola JT, Wekerle H, Lees MB: Monoclonal antibodies against myelin proteolipid protein: identification and characterization of two major determinants. J Neurochem 1991;57:1671-1680.

14 Iai M, Yamamura T, Takashima S: Early expression of proteolipid protein in human fetal and infantile cerebri. Pediatr Neurol 1997;17:235-239.

15 Smith PK, Krohn RI, Hermanson GT, Mallia AK, Gartner FH, Provenzano MD, Fujimoto EK, Goeke NM, Olson BJ, Klenk DC: Measurement of protein using bicinchoninic acid. Anal Biochem 1985;150:76-85.

$\checkmark 16$ Williams RD, Wang E, Merrill AH: Enzymology of long-chain base synthesis by liver: characterization of serine palmitoyltransferase in rat liver microsomes. Arch Biochem Biophys 1984;228:282-291.

17 Morell P, Radin NS: Synthesis of cerebroside by brain from uridine diphosphate galactose and ceramide containing hydroxy fatty acid. Biochemistry 1969;8:506-512.
18 Cuzner ML, Davison AN: The lipid composition of rat brain myelin and subcellular fractions during development. J Biochem 1968; 106:29-34

19 O’Brien JS, Sampson EL: Lipid composition of the normal human brain: gray matter, white matter, and myelin. J Lipid Res 1965;6: 537-544

20 Davison AN, Cuzner ML, Banik NL, Oxberry J: Myelinogenesis in the rat brain. Nature 1966;212:1373-1374.

21 Wells MA, Dittmer JC: A microanalytical technique for the quantitative determination of twenty-four classes of brain lipids. Biochemistry 1966;5:3405-3418.

22 Johnston M, Silverstein F: Perinatal anoxia; in Coyle JT (ed): Animal Models of Dementia. New York, Alan R Liss, 1987, pp 223-251.

23 Spandou E, Papadopoulou Z, Soubasi V, Karkavelas G, Simeonidou C, Pazaiti A, Guiba-Tziampiri O: Erythropoietin prevents long-term sensorimotor deficits and brain injury following neonatal hypoxia-ischemia in rats. Brain Res 2005;1045:22-30.

24 Adair J, Filloux F: Effects of hypoxic-ischemic brain damage on dopaminergic markers in the neonatal rat: a regional autoradiographic analysis. J Child Neurol 1992;7: 199-207.

25 Burke RE, Karanas AL: Quantitative morphological analysis of striatal cholinergic neurons in perinatal asphyxia. Ann Neurol 1990;27:81-88.

26 Gibney SM, McDermott KW: Differentiation of oligodendrocytes in neurospheres derived from embryonic rat brain using growth and differentiation factors. J Neurosci Res 2007;85:1912-1920. 